\title{
Comparative immunohistochemical study of deep infiltrating endometriosis, lymph node endometriosis and atypical ovarian endometriosis including description of a perineural invasion
}

\author{
Jiri Lenz ${ }^{\mathrm{a}, \mathrm{b}, \mathrm{c}}$, Radek Chvatal ${ }^{\mathrm{d}}$, Ludek Fiala ${ }^{\mathrm{e}, \mathrm{f}}$, Petra Konecna ${ }^{\mathrm{c}}$, David Lenz ${ }^{\mathrm{b}}$
}

\begin{abstract}
Aim. Endometriosis is an inflammatory condition that shares a number of similarities with malignant diseases, such as an abnormal morphology, migration along the nerve bundles and metastatic spread to lymph nodes and distant organs. Endometriotic lesions are associated with oestrogen and progesterone imbalance which seems to play a key role in the pathogenesis of endometriosis. The aim of this study was to compare the status of both oestrogen and progesterone receptors in tissue of deep infiltrating endometriosis, lymph node endometriosis and atypical ovarian endometriosis using immunohistochemical methods, as well as to investigate the relationship between endometriosis and protein p53. Methods. A total of 40 cases with deep infiltrating endometriosis were included in our study. Based on histopathological analysis of resected specimens, the cases were divided into 2 groups: group 1 - lymph node endometriosis (cases with lymph node involvement; $n=12$ ) and group 2 - deep infiltrating endometriosis (cases without lymph node involvement; $\mathrm{n}=28$ ). As a control group, eutopic endometrium of adenomyosis- and endometriosis-free women were used $(n=16)$. Five cases of atypical ovarian endometriosis as well as descriptions of the nerve involvement in endometriosis were also included. Immunohistochemical staining with a total of 4 markers was performed - oestrogen and progesterone receptors (ER, PR), p53 and Ki-67 (proliferation index).
\end{abstract}

Results. The immunophenotype of the cases in groups 1 and 2 and in the control group was virtually identical in the proliferative phase - strong nuclear ER and PR expression in more than $90 \%$ of endometrial glandular and stromal cells. In the early and mid secretory phase, ER expression only slightly decreased $(80 \%)$ in endometrial glandular cells in group 2 and the control group, whereas in the late secretory phase, significant decrease of ER expression only in the control group was observed (15-50\%; $P<0.001)$. In group 2 and the control group, significant decrease of PR expression only in endometrial glandular cells was observed in the mid and late secretory phase (less than $15 \% ; P<0.001$ ). Differences in receptor content were found only in isolated cases in group 2. In group 1, no secretory changes were found. In all three groups, sporadic and weak nuclear p53 expression in less than $3 \%$ in both endometrial glandular and stromal cells was detected (regardless of the phase of the menstrual cycle). In atypical ovarian endometriosis, higher and strong p53 expression (on average 26\%) and decrease in ER (on average 56\%) and PR (less than 1\%) expression was observed; compared to the control group and groups 1 and 2, the differences for all 3 markers were highly significant $(P<0.001)$. In all groups, the proliferation index (Ki-67) reached the highest values in the proliferation phase and decreased during the cycle. However, in endometriotic tissue, it was widely variable in the individual phases of the cycle. Perineural spread of endometriosis with significant neural hypertrophy, hyperplasia and involvement of the ganglia of the autonomic nervous system was detected in 5 cases (12.5\%).

Conlusion. From a histological and immunohistochemical point of view, deep infiltrating endometriosis and lymph node endometriosis appear to represent the same entity. For the first time, a simple immunohistochemical panel with antibodies against ER, PR and p53 useful in diagnosing atypical endometriosis has been described. The marked endometriosis-associated neural changes (endometriotic neuropathy) could be one of the causes of impaired function of the affected organs after debulking surgery with macroscopic negative resection margins as well as pain symptomatology in macroscopic inapparent endometriotic lesions.

Key words: deep infiltrating endometriosis, lymph node endometriosis, atypical endometriosis, immunohistochemistry, p53, perineural invasion

Received: August 13, 2019; Revised: February 12, 2020; Accepted: February 14, 2020; Available online: March 9, 2020 https://doi.org/10.5507/bp.2020.006

(c) 2021 The Authors; https://creativecommons.org/licenses/by/4.0/

${ }^{a}$ Department of Pathology, Znojmo Hospital, Czech Republic

${ }^{b}$ Cytohisto s.r.o., Breclav, Czech Republic

'Department of Anatomy, Histology and Embryology, Faculty of Veterinary Medicine, University of Veterinary and Pharmaceutical Sciences Brno, Czech Republic

${ }^{d}$ Department of Obstetrics and Gynaecology, Znojmo Hospital, Czech Republic

eObstetrics and Gynaecology Private Clinic, Bulovka Hospital, Czech republic

fInstitute of Sexology, 1st Faculty of Medicine, Charles University, Prague, Czech Republic

Corresponding author: Jiri Lenz, e-mail:jiri.lenz@gmail.com 


\section{INTRODUCTION}

Endometriosis is a common, oestrogen-dependent, inflammatory disease associated with infertility and pelvic pain. It is defined as the presence of endometrium outside the uterus ${ }^{1}$.

The endometriosis classification of the revised American Society for reproductive Medicine (rASRM) differentiates between deep infiltratitng endometriosis (DIE), peritoneal endometriosis and ovarian endometriosis ${ }^{2}$. Diagnostic criteria for DIE have been proposed - either they are lesions that affect the intestine, urinary bladder, ureter, vagina or uterosacral ligaments or lesions that extend more than $5 \mathrm{~mm}$ below the peritoneal surface ${ }^{3}$. DIE represents a subset of endometriosis with an ill-defined set of symptoms and lack of specific diagnostic criteria which frequently cause significant delays in treatment ${ }^{4}$.

Endometriosis is traditionally considered a benign disease. However, some features that are typical of malignant disease have been identified, including metastatic spread to lymph nodes and distant organs, abnormal morphology, migration along the nerve bundles, deregulated cell growth, cellular invasion, neoangiogenesis, DNA aneuploidy and loss of heterozygosity ${ }^{5}$.

Lymph node involvement due to endometriosis is uncommon, but it has been documented as a frequent event (25-42\%) in bowel, rectosigmoid and rectovaginal endometriosis ${ }^{6-8}$. Microscopically, two stages of lymph node endometriosis (LNE) are distinguishable - isolated endometriotic-like cells (early stage) and metastatic endometriotic lesions (late stage) (ref. ${ }^{7}$ ).

A variety of hyperplastic and atypically hyperplastic changes similar to those occurring in the eutopic endometrium may appear in endometriotic lesions ${ }^{9}$. In view of this finding, the term atypical endometriosis was introduced.

The pathogenesis of endometriosis is not entirely clear. However, oestrogen and progesterone imbalance seems to play a key role in the migration, proliferation and infiltration of the peritoneum by endometrial cells ${ }^{10}$. Hormonal imbalance in endometriosis also represents a target in the treatment of the disease ${ }^{11}$.

The main goal of this study was to determine and compare the status of both oestrogen and progesterone receptors in a tissue of DIE, LNE and atypical ovarian endometriosis using immunohistochemical methods.

The possible relationship between endometriosis and protein p53 was also investigated.

The p53 molecule is a tumor suppressor that prevents the outgrowth of aberrant cells by inducing cell cycle arrest, DNA repair, or programmed cell death ${ }^{12}$. Its commonly mutated in several human cancers, but p53 accumulation may also occur in non-tumour cells under various forms of stress (e.g. in a variety of autoimmune diseases) (ref. $\left.{ }^{13}\right)$. Staining is associated with aneuploidy, increased $\mathrm{S}$ phase fraction and genetic instability ${ }^{14}$.

\section{MATERIALS AND METHODS}

\section{Study group and tissue specimens}

A total of 40 patients with DIE were included in our study, the mean age was 33.1 years (range 22.0 - 47.0); ten patients were cigarette smokers (25\%) (Table 1). All patients underwent surgery for DIE at the centre for the treatment of endometriosis at Znojmo Hospital between 2016-2018. The nature of the surgery varied widely depending on the extent of the disease. A minimally invasive laparoscopic approach was always preferred, but in 10 cases, conversion to laparotomy was neccesary. For classification of endometriosis rASRM score and ENZIAN classification were used.

All patients were premenopausal, knowledge of the date of the last menstrual period and the length of the menstrual cycle was derived from the available anamnestic data. Nine of the 40 patients received hormonal therapy at the time of surgery, 8 patients took progestincontaining medication (treatment for endometriosis, dysfunctional uterine bleeding or birth control) and 1 patient took gonadotropin releasing hormone analogue (GnRH analogue; treatment for endometriosis). The rest had not received hormonal therapy for at lest 4 months before surgery.

Histological examination confirmed a diagnosis of DIE in all selected cases. Based on histopathological analysis of resected specimens, the cases were divided into 2 groups: group 1 - lymph node endometriosis (cases with lymph node involvement; $n=12$ ) and group 2 - deep infiltrating endometriosis (cases without lymph node involvement; $n=28$ ). Cases with florid fibrosis, inflammatory or regressive changes were not included in the study. Five cases of atypical ovarian endometriosis were also included in our study. As a control group, eutopic endometrium of adenomyosis- and endometriosis-free women was used $(n=16)$. Uterine samples were obtained from normally cycling women undergoing hysterectomy for cervical intraepithelial neoplasia $(n=8)$, leiomyomata $(n=4)$ or chronic pelvic pain $(n=4)$.

The diagnosis of endometriosis was based on the presence of both endometriotic glands and stroma while diagnosis of atypical endometriosis was based on histopathological criteria reported by LaGrenade and Silverberg ${ }^{15}$ and Czernobilsky and Morris ${ }^{16}$. These criteria include eosinophilic cytoplasm, large hyperchromatic or pale nuclei with moderate to marked pleomorphism, an increased nuclear to cytoplasmic ratio, cellular crowding and stratification or tufting. Three or more of these criteria were present in all cases (Fig. 1a).

Hematoxylin and eosin stained sections of endometrial samples were dated by the criteria described by Noyes et al. ${ }^{17}$. As in eutopic endometrium, the histologic appearance of the endometrial epithelium and stroma in endometriotic lesions was classified as early, mid and late proliferative and early, mid and late secretory. Some cases with early proliferative morphology have partly acquired an inactive appearance (characterised by small glands lined by low columnar epithelium with minimal mitotic activity). 
Table 1. Descriptive statistics of study population including pathologic features of deep infiltrating endometriosis and immunohistochemical analysis of oestrogen and progesterone receptor expression in deep infiltrating endometriosis (group 2).

\begin{tabular}{|c|c|c|c|c|c|c|c|c|}
\hline Case & Age & Medications & $\begin{array}{l}\text { Site } \\
\text { of endo- } \\
\text { metriosis }\end{array}$ & $\begin{array}{l}\text { Lymph node } \\
\text { involvement }\end{array}$ & $\begin{array}{c}\text { Day } \\
\text { of cycle }\end{array}$ & $\begin{array}{l}\text { Histologic } \\
\text { appearance of } \\
\text { deep infiltrating } \\
\text { endometriosis }\end{array}$ & $\begin{array}{c}\text { ER } \\
\text { expression* }\end{array}$ & $\begin{array}{c}\text { PR } \\
\text { expression* }\end{array}$ \\
\hline 1 & 30 & GnRH analogue & Bladder & + & 58 & EP & 100 & 100 \\
\hline $2 \uparrow$ & 37 & None & Vagina & - & 10 & MP & 100 & 100 \\
\hline 3 & 45 & Progestins & Sigmoideum & - & 5 & A & 90 & 0 \\
\hline 4 & 28 & None & USL & - & 24 & LS & 90 & 15 \\
\hline $5 \dagger$ & 35 & None & Sigmoideum & + & 11 & MP & 100 & 100 \\
\hline 6 & 40 & Progestins & USL & - & 37 & I & 100 & 100 \\
\hline 7 & 29 & None & Rectum & + & 10 & MP & 100 & 100 \\
\hline $8 \dagger$ & 32 & None & Rectum & - & 24 & MP & 100 & 100 \\
\hline 9 & 33 & None & USL & - & 43 & EP & 100 & 100 \\
\hline 10 & 22 & Progestins & USL & - & 11 & I & 100 & 100 \\
\hline 11 & 27 & Progestins & USL & - & 30 & I & 100 & 100 \\
\hline 12 & 43 & Progestins & USL & - & 31 & I & 100 & 100 \\
\hline 13 & 29 & None & USL & - & 14 & I & 40 & 70 \\
\hline 14 & 41 & None & USL & - & 22 & I & 90 & 80 \\
\hline $15 \dagger$ & 34 & None & USL & + & 8 & EP & 100 & 100 \\
\hline 16 & 26 & None & USL & + & 8 & I & 100 & 100 \\
\hline 17 & 24 & None & Bladder & + & 6 & EP & 90 & 70 \\
\hline 18 & 27 & None & USL & - & 14 & I & 100 & 100 \\
\hline 19 & 41 & None & USL & - & 15 & I & 90 & 90 \\
\hline $20 \dagger$ & 29 & None & Vagina & - & 18 & EP & 100 & 100 \\
\hline 21 & 25 & None & Vagina & - & 20 & LP & 100 & 100 \\
\hline 22 & 28 & None & Vagina & - & 32 & MS & 80 & 1 \\
\hline 23 & 25 & None & Bladder & - & 21 & MS & 90 & 5 \\
\hline 24 & 28 & Progestins & Bladder & - & 45 & A & 80 & 1 \\
\hline 25 & 35 & None & Vagina & + & 5 & MP & 100 & 100 \\
\hline 26 & 42 & None & Bowel & - & Unknown & $\mathrm{C}$ & 90 & 5 \\
\hline 27 & 25 & None & USL & + & 3 & I & 100 & 100 \\
\hline 28 & 37 & None & USL & - & 14 & I & 100 & 100 \\
\hline 29 & 35 & None & USL & - & 20 & $\mathrm{C}$ & 90 & 10 \\
\hline 30 & 36 & None & USL & - & 10 & MP & 100 & 100 \\
\hline 31 & 41 & None & USL & - & 7 & I & 100 & 100 \\
\hline 32 & 24 & None & Rectum & + & 5 & EP & 100 & 100 \\
\hline 33 & 26 & Progestins & Rectum & + & 11 & I & 100 & 100 \\
\hline 34 & 35 & None & USL & - & 4 & I & 100 & 100 \\
\hline 35 & 28 & None & USL & - & 5 & EP & 100 & 100 \\
\hline 36 & 42 & None & Sigmoideum & + & 24 & EP & 90 & 100 \\
\hline 37 & 40 & None & USL & - & 17 & EP & 100 & 100 \\
\hline 38 & 31 & None & Rectum & + & 15 & MP & 100 & 100 \\
\hline 39 & 47 & Progestins & Bladder & - & 48 & I & 100 & 100 \\
\hline 40 & 33 & None & USL & - & 8 & EP & 100 & 100 \\
\hline
\end{tabular}

* Percentage (\%) of marker positive endometrial glandular cells.

$\uparrow$ Cases with nerve involvement by endometrios.

A, atrophy with weak secretory changes; $\mathrm{C}$, a combination of proliferative and secretory endometrium; $\mathrm{EP}$, early proliferative; ER, oestrogen receptor; GnRH, gonadotropin releasing hormone; LP, late proliferative; LS, late scretory; MP, mid proliferative; MS, mid secretory; PR, progesterone receptor; USL, uterosacral ligament.

Routinely processed formalin fixed and paraffin embedded tissues were available for immunohistochemical analysis in all cases. The tissue specimens, slides, and histopathology reports were retrieved from the files of the department of pathology. All resection specimens were fixed in $10 \%$ neutral buffered formalin for $24 \mathrm{~h}$. The $4 \mu \mathrm{m}$ thick tissue sections stained with hematoxylin-eosin were reviewed by pathologist and representative samples with structures of deep infiltrating endometriosis, lymph node endometriosis and atypical ovarian endometriosis were selected for immunohistochemical analysis.

The study was approved by the ethics comittee of 

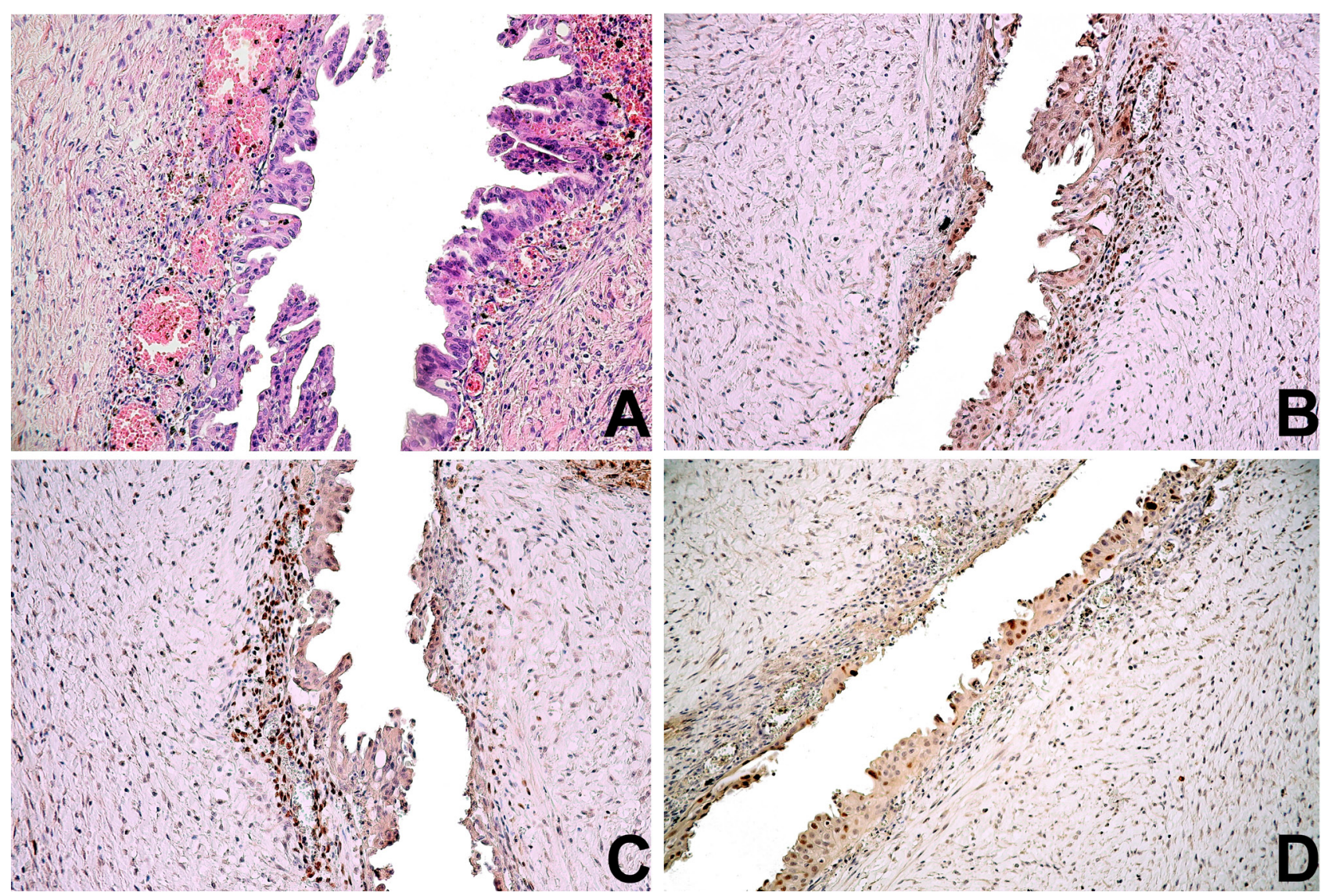

Fig. 1. Atypical ovarian endometriosis and immunohistochemical expression of ER, PR and p53 in atypical ovarian endometriosis. A: Atypical ovarian endometriosis consisting of cells with eosinophilic cytoplasm, large hyperchromatic or pale nuclei with moderate to marked pleomorphism, an increased nuclear to cytoplasmic ratio, cellular crowding, stratification and tufting. Note the absence of the acute inflammatory infiltrate (hematoxylin-eosin staining, original magnification $\times 200$ ).

B: Nuclear expression of ER in approximately $40 \%$ of endometrial glandular cells with only moderately staining (immunohistochemistry, original magnification $\times 200$ ).

C: Nuclear expression of PR in less than $1 \%$ of endometrial glandular cells (immunohistochemistry, original magnification $\times 200$ ). D: Strong nuclear expression of p53 in approximately $28 \%$ of endometrial glandular cells (immunohistochemistry, original magnification $\times 200$ ).

Znojmo Hospital. The requirement for patient consent was waived becasuse of the retrospective nature of this study.

\section{Immunohistochemistry}

Immunohistochemistry was performed for all cases, using antibodies against oestrogen receptors (clone ER SP1, Roche, RTU), progesterone receptors (clone PR 1E2, Roche, RTU), p53 (clone Bp53-11, Roche, RTU) and Ki-67 (clone 30-9, Roche, RTU). All immunohistochemical staining was performed on the automated immunostainer BenchMark GX (Ventana Medical System, Inc., Tucson, AZ, USA); ultraView Universal DAB Detection Kit was used.

The primary antibodies were visualised using hydrogen peroxide substrate and 3, 3'- diaminobenzidine tetrahydrochloride (DAB) chromogen. External positive controls were used for all antibodies (invasive breast carcinoma for ER and PR, tonsil for p53 and lymph node for Ki-67). Negative controls were prepared by incubating samples without a primary antibody. Evaluation of immunohistochemical results was performed using a uniform micro- scope and camera setting (Olympus BX43F microscope and Promican 3-3CC camera).

\section{Evaluation of immunostaining}

In the endometrial tissue, nuclear immunostaining for ER, PR, p53 and Ki-67 was regarded as positive. The percentages of marker positive cells were evaluated using a light microscope at $\times 200$ magnification. At least five foci of eutopic/ectopic endometrium were analyzed and the percentage of marker positive cells of the covered area was determined. The intensity of immunoreaction with antibodies against ER, PR and p53 was also investigated; weak, moderately and strong staining was distinguished.

\section{Statistical analysis}

Standard descriptive statistics were used to summarise the data. Continuous variables were expressed as means and standard deviation, and statistically significant differences (at the level of $\alpha=0.05$ ) between means were evaluated using the non-parametric Mann-Whitney rank sum test with continuity correction where appropriate. 
Table 2. Pathologic features of lymph node endometriosis and immunohistochemical analysis of oestrogen and progesterone receptor and p53 expression in lymph node endometriosis (group 1) and atypical ovarian endometriosis.

\begin{tabular}{|c|c|c|c|c|c|c|c|c|}
\hline \multirow[b]{2}{*}{ Case } & \multicolumn{4}{|c|}{$\begin{array}{l}\text { Lymph node } \\
\text { endometriosis }\end{array}$} & \multicolumn{4}{|c|}{$\begin{array}{l}\text { Atypical ovarian } \\
\text { endometriosis }\end{array}$} \\
\hline & $\begin{array}{l}\text { Histologic } \\
\text { appearance } \\
\text { of lymh node } \\
\text { endometriosis }\end{array}$ & $\begin{array}{c}\text { ER } \\
\text { expression* }\end{array}$ & $\begin{array}{c}\text { PR } \\
\text { expression* }\end{array}$ & $\begin{array}{c}\text { p53 } \\
\text { expression* }\end{array}$ & Case & $\begin{array}{c}\text { ER } \\
\text { expression* }\end{array}$ & $\begin{array}{c}\text { PR } \\
\text { expression* }\end{array}$ & $\begin{array}{c}\mathrm{p} 53 \\
\text { expression* }\end{array}$ \\
\hline 1 & EP & 100 & 95 & 1 & 1 & 40 & 1 & 20 \\
\hline 2 & EP & 100 & 100 & 1 & 2 & 55 & 0 & 26 \\
\hline $3 \dagger$ & A & 90 & 90 & 0 & 3 & 70 & 0 & 27 \\
\hline 4 & A & 95 & 95 & 1 & 4 & 55 & 1 & 28 \\
\hline 5 & EP & 90 & 90 & 0 & 5 & 60 & 0 & 27 \\
\hline 6 & EP & 100 & 95 & 0 & - & - & - & - \\
\hline 7 & EP & 90 & 95 & 1 & - & - & - & - \\
\hline 8 & EP & 95 & 90 & 0 & - & - & - & - \\
\hline 9 & A & 90 & 90 & 1 & - & - & - & - \\
\hline $10 \dagger$ & EP & 100 & 90 & 1 & - & - & - & - \\
\hline 11 & EP & 95 & 95 & 0 & - & - & - & - \\
\hline 12 & EP & 100 & 90 & 0 & - & - & - & - \\
\hline
\end{tabular}

* Percentage (\%) of marker positive endometrial glandular cells.

$\dagger$ Involvement of 2 lymph nodes by endometrios.

A, atrophic; EP, early proliferative; ER, oestrogen receptor; PR, progesterone receptor.

\section{RESULTS}

DIE was diagnosed in all 40 patients with incidentally removed lymph nodes. In our series, which was a total of 118 coincidentally resected lymph nodes; the endometriotic lesions were noted in 15 of them (12.7\%).

\section{Pathologic features of lymph node endometriosis (group 1) (Table 2)}

All cases of LNE consisted of both endometrial glandular and stromal elements located in the lymph node capsule or cortex. The glands were mostly separated, focally cystic dilated, occasionally formed papillary structures and in one case surrounded by a lesser amount of hypocelular dense fibrous stroma. No microcalcification or psammoma bodies were found. The endometriotic glands were lined with weak proliferative endometrial epithelium with mild cellular atypia (early proliferative appearance), focal ciliated metaplasia and low mitotic activity; secretory changes were not found. Only in the case of stromal fibrosis did the epithelium seem to be flattened (atrophic). Haemosiderin-laden macrophages and pseudoxanthoma cells were a common finding in the stroma (Fig. 2a-c).

\section{Pathologic features of deep infiltrating endometriosis (group 2) (Table 1)}

The predominant pattern of the endometriotic tissue was early proliferative (10 cases, $25 \%$ ) or inactive (15 cases, $37.5 \%)$. The others were classified as mid proliferative ( 7 cases, $17.5 \%$ ), late proliferative ( 1 case, $2.5 \%$ ), mid secretory ( 2 cases, $5 \%$ ) and late secretory ( 1 case, $2.5 \%$ ). In two cases, a combination of proliferative and secretory endometrium was found (5\%). In 9 patients receiving hormonal therapy, the inactive pattern was found in 7 cases, while the atrophic pattern with weak secretory changes was found in 2 cases (5\%).

When more than one endometriotic focus was examined in the same patient, the appearance of the specimens did not differ significantly.

The appearance of endometriotic tissue was synchronous with the dating of the menstrual cycle in 11 cases $(27.5 \%)$ - early proliferative (5 cases), mid proliferative (4 cases), mid secretory ( 1 case) and late secretory (1 case).

The most common changes in DIE were ciliated (tubal) metaplasia, cystic changes and focal endometrial hyperplasia without atypia. Cystic changes were found in 19 cases (47.5\%), endometrial hyperplasia without atypia in 2 cases (5\%) and a combination of both in 6 cases (15\%). In a total of 13 cases (32.5\%), no structural changes were found. Endometrial hyperplasia was characterised by outpouching and dilatation of glandular epithelium (simple hyperplasia), but with an increasing degree of architectural abnormality, glands become complex, branched, crowded or back-to-back (complex hyperplasia). The lining cells were columnar, mostly stratified, with oval or elongated bland nuclei with smooth contours, fine even chromatine and variable mitotic activity (no more than seven mitotic figures per $10 \mathrm{high}$-power fields). Only in 1 case $(2.5 \%)$, was mucinous metaplasia found.

Eutopic endometrium was available in 6 cases of DIE. In 4 (67\%), the histologic appearance of endometriotic tissue was synchronous with the corresponding eutopic endometrium.

Perineural and intraneural invasion (PNI) was detect- 

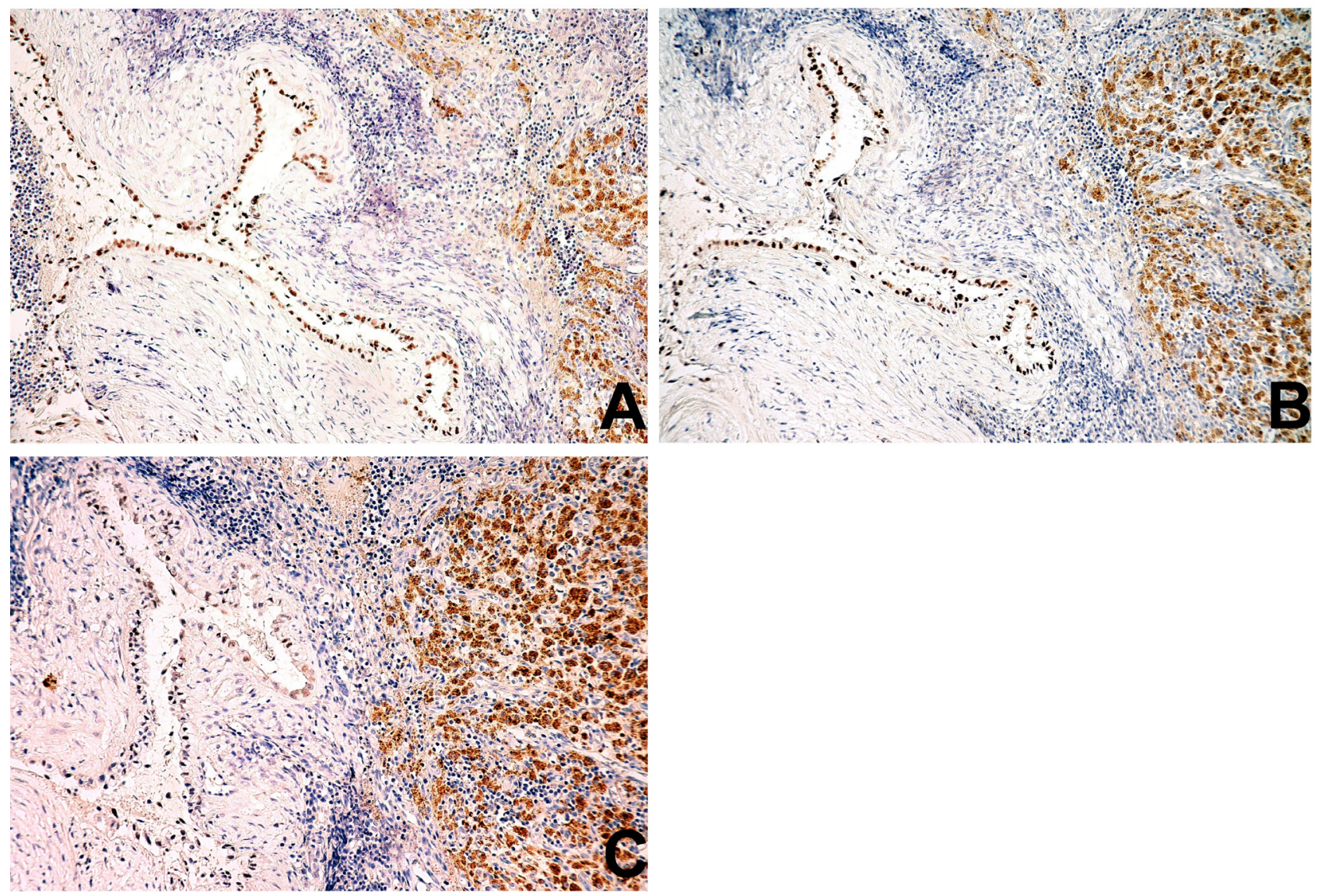

Fig. 2. Immunohistochemical expression of ER, PR and p53 in lymph node endometriosis.

A: Strong nuclear expression of ER in more than $90 \%$ of endometrial glandular cells. Periglandular fibrosis, haemosiderin-laden macrophages and pseudoxanthoma cells are noted (immunohistochemistry, original magnification $\times 200$ ).

B: Strong nuclear expression of PR in more than $90 \%$ of endometrial glandular cells (immunohistochemistry, original magnification $\times 200$ ).

C: Weak nuclear expression of p53 in less than $1 \%$ of endometrial glandular cells (immunohistochemistry, original magnification $\times 200)$.
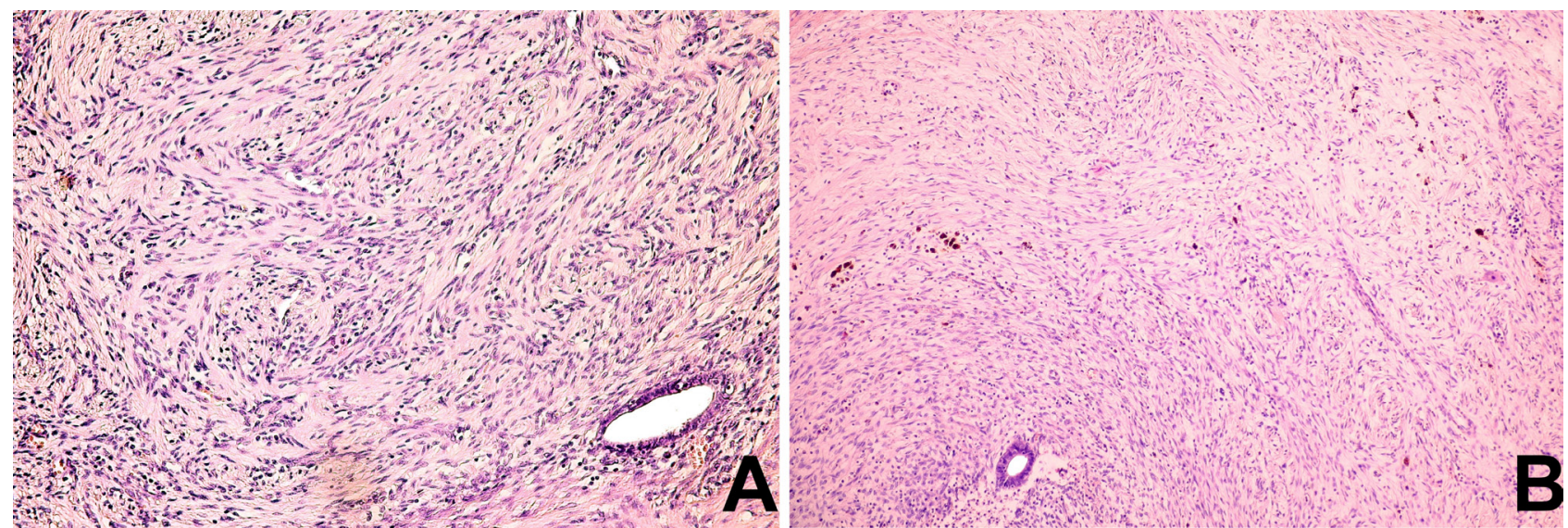

Fig. 3. Endometriotic neuropathy.

A: Intraneural spread of endometriotic gland (visible in the lower right corner) with marked neural hypertrophy and hyperplasia (hematoxylin-eosin staining, original magnification $\times 100$ ).

B: Involvement of the parasympathetic ganglion of the autonomic nervous system by endometriosis. Numerous bundles of nerve fibers, cell body of the postsynaptic neuron (visible in the right half of the field) and deposits of haemosiderin are noted (hematoxylin-eosin staining, original magnification $\times 100$ ). 
ed in a total of 5 cases $(12.5 \%)$ - rectal endometriosis ( 1 case), rectosigmoid endometriosis ( 1 case), vaginal endometriosis ( 2 cases) and endometriosis of the uterosacral ligament ( 1 case). The nerve involvement due to endometriosis occurred in the organ (bowel or vaginal) wall and also in the adjacent tissue. PNI was a multifocal finding with concomitant marked neural hypertrophy and hyperplasia (Fig. 3a). An interesting finding was the involvement of the parasympathetic (terminal) ganglia located in, or close to, the wall of the affected organs (Fig. 3b).

\section{Analysis of ER, PR, Ki-67 and p53 expression in the control group (Table 3 )}

Expression of ER in glands of normal endometrium was maximal in the proliferation phase (more than 90\%), slightly decreased in the early and mid secretory phase (about 80\%) and lowest being in the late secretory phase (15-50\%). Expression of PR was identical in the proliferative and early secretory phase, a significant decrease occurred in the mid secretory (less than 10\%) and the late secretory phase (less than $1 \% ; P<0.001$ ). For both types of hormonal receptors, expression in endometrial stromal cells was high, regardless of the phase of the menstrual cycle $(90 \%)$. The intensity of staining in both endometrial glands and stroma for ER and PR was moderate or strong.

The proliferation index (Ki-67) in glands also showed a decrease during the cycle, the highest values being reached in the mid proliferative phase $(50-90 \%$, on average $70 \%$ ), lower in the late proliferation phase $(1-40 \%$, on average $22 \%$ ), while a significant decrease with minimal expression was observed in mid and late secretory phase (about $1 \% ; P<0.001$ ).

Weak nuclear expression of p53 in less than $3 \%$ in both type of cells was detected.

\section{Analysis of ER, PR, Ki-67 and p53 expression \\ in the lymph node endometriosis (group 1) (Table 2) and deep infiltratring endometriosis (group 2) (Table 1)}

The results of immunohistochemical staining with antibodies against ER, PR, Ki-67 and p53 were somewhat similar to those in the control group. In both groups, strong nuclear expression of ER and PR in more than $90 \%$ of cells in both endometrial glands and stroma in the proliferative phase was observed (i.e. there were no differences between LNE and DIE in the proliferative phase) (Fig. 2a,b and Fig. 4a,c). Only in one case in group 2 , decrease in the PR expression only in glandular cells (70\%) was found. In group 2, in the mid and late secretory phase, ER expression only slightly decreased (to more than $80 \%$ ) (Fig. 4b), whereas a significant decrease of $\mathrm{PR}$ in endometrial glands was detected (on average $7 \%$; $P<0.001$ ) (Fig. 4c).

In group 1, all cases with atrophic morphology showed expression of ER and PR in more than $90 \%$ of glandular and stromal cells.

In both groups, Ki-67 expression in endometrial glands was quite variable. In the early proliferative phase it ranged between 1 and 50\% (on average 34.2\% for both groups), in the mid proliferative phase it ranged between 10 and $70 \%$ (on average $44 \%$ only for group 2 ) and in the late proliferative phase it was $10 \%$ (only group 2 ); no

Table 3. Immunohistochemical analysis of oestrogen and progesterone receptor, p53 and Ki-67 expression in eutopic endometrium (control group).

\begin{tabular}{ccccccc}
\hline Case & Day of cycle & Stage of cycle & $\begin{array}{c}\text { ER } \\
\text { expression* }\end{array}$ & $\begin{array}{c}\text { PR } \\
\text { expression* }\end{array}$ & $\begin{array}{c}\text { p53 } \\
\text { expression* }\end{array}$ & $\begin{array}{c}\text { Ki-67 } \\
\text { expression* }\end{array}$ \\
\hline 1 & 6 & EP & 100 & 100 & 0 & 40 \\
2 & 8 & EP & 100 & 100 & 2 & 60 \\
3 & 10 & MP & 100 & 100 & 1 & 70 \\
4 & 9 & MP & 100 & 95 & 3 & 50 \\
5 & 9 & MP & 100 & 100 & 0 & 70 \\
6 & 11 & MP & 100 & 100 & 3 & 1 \\
7 & 13 & LP & 95 & 90 & 1 & 1 \\
8 & 13 & LP & 90 & 90 & 100 & 40 \\
9 & 12 & LP & 100 & 90 & 1 & 10 \\
11 & 16 & ES & 90 & 100 & 1 & 30 \\
12 & 18 & ES & 100 & 10 & 0 & 25 \\
13 & 22 & MS & 80 & 2 & 0 & 1 \\
14 & 20 & MS & 80 & 1 & 0 & 1 \\
16 & 19 & MS & 75 & 0 & 0 & 1 \\
\end{tabular}

\footnotetext{
* Percentage (\%) of marker positive endometrial glandular cells.
}

EP, early proliferative; ER, oestrogen receptor; ES, early secretory; LP, late proliferative; LS, late secretory; MP, mid proliferative; MS, mid secretory; PR, progesterone receptor. 

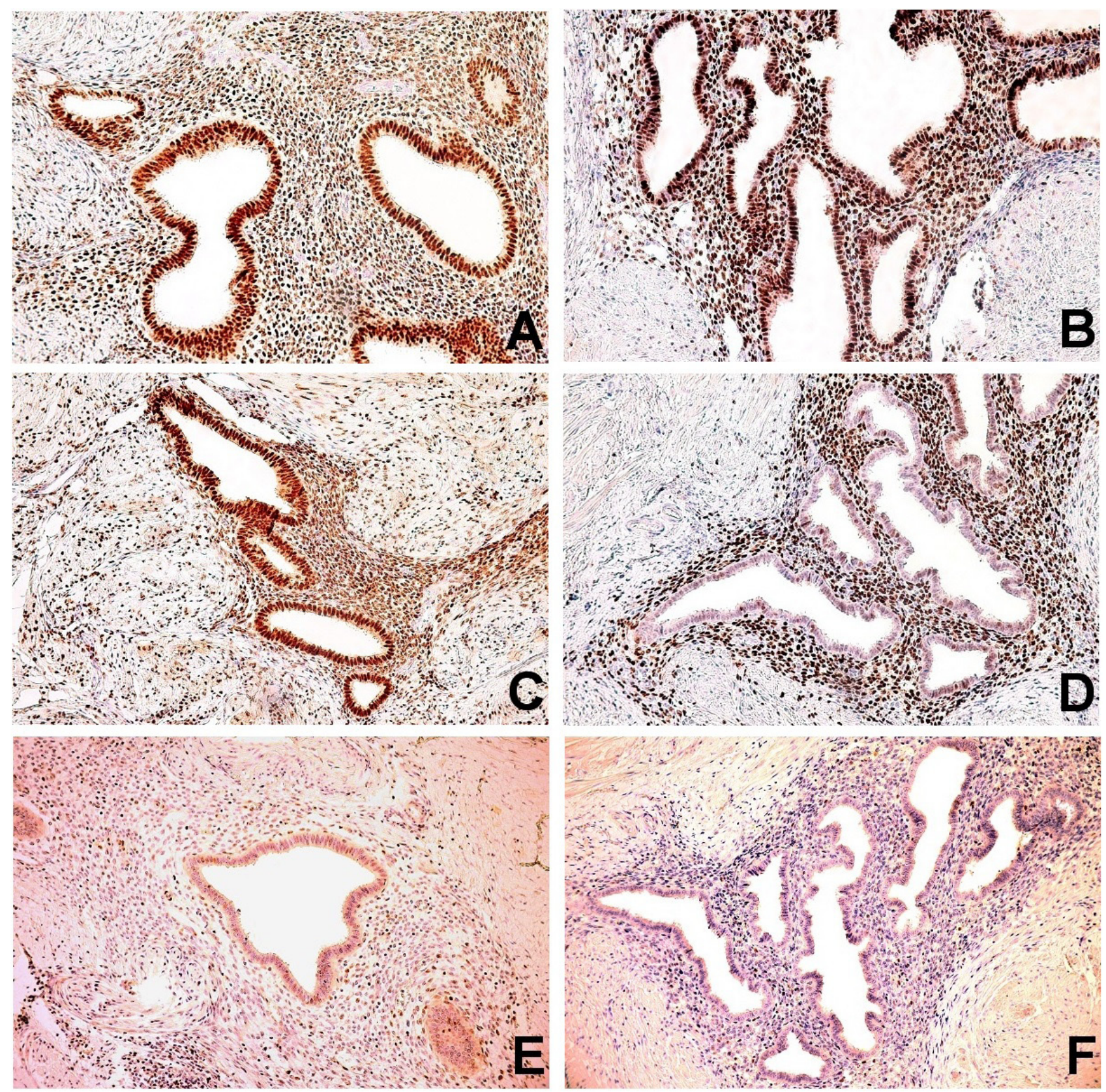

Fig. 4. Immunohistochemical expression of ER, PR and p53 in deep infiltrating endometriosis in the mid proliferative and mid secretory phase of the cycle.

A: Diffuse strong nuclear expression of ER in both endometrial glands and stroma in the mid proliferative phase of the cycle (immunohistochemistry, original magnification $\times 200$ ).

B: Diffuse strong nuclear expression of ER in both endometrial glands and stroma in the mid secretory phase of the cycle (immunohistochemistry, original magnification $\times 200$ ).

C: Diffuse strong nuclear expression of PR in both endometrial glands and stroma in the mid proliferative phase of the cycle (immunohistochemistry, original magnification $\times 200$ ).

D: Loss of PR expression in endometrial glands but not in endometrial stroma in the mid secretory phase of the cycle (immunohistochemistry, original magnification $\times 200$ ).

E: Absence of p53 expression in both endometrial glands and stroma in the mid proliferative phase of the cycle (immunohistochemistry, original magnification $\times 200$ ).

F: Absence of $\mathrm{p} 53$ expression in both endometrial glands and stroma in the mid secretory phase of the cycle (immunohistochemistry, original magnification $\times 200$ ).

differences between LNE and DIE in the proliferative phase were found (in group 1, no secretory changes were found). As in the control group, a significant decrease in the secretory phase in group 2 was observed (less than $15 \% ; P<0.001)$.
In both groups, weak and sporadic nuclear p 53 expression in less than $1 \%$ in endometrial glands and stroma was detected (Fig. 2c and Fig. 4e,f).

In group 2, the immunophenotype of inactive appearing glands was as follows: strong nuclear expression of 
both ER and PR in more than $90 \%$ of cells, minimal Ki-67 expression (less than $1 \%$ ) and absence of p53 expression $(0 \%)$. In one case, significant decrease in ER expression (40\%), and in two cases a decrease in PR expression (70 and $80 \%$ ) was observed.

In group 2, in the two cases of atrophic pattern with secretory changes, ER expression was observed in more than $80 \%$ of glandular cells, while PR expression was almost lost (less than 1\%). In endometrial stromal cells, high expression of both hormonal receptors was detected (more than $80 \%$ ).

In the remaining two cases in group 2 (a combination of proliferative and secretory endometrium) ,secretory pattern" of hormonal receptor expression in endometriotic glands was observed (high ER expression and very low PR expression).

\section{Analysis of ER, PR, p53 and Ki-67 in atypical ovarian endometriosis (Table 2)}

A different immunophenotype in all 5 cases of atypical ovarian endometriosis was found. Compared to the control group and groups 1 and 2, differences were related to ER, PR and p53. Expression of ER was on average $56 \%$ (range 40-70\%) (Fig. 1b), whereas expression of PR was less than 1\% (Fig. 1c). Differences for both hormonal receptors were statistically significant $(P<0.001)$; the staining was moderately or strong.

Only in the lesions of atypical ovarian endometriosis was a strong nuclear p53 expression detected. The number of positive glandular cells ranged between 20 and $28 \%$ (on average 26\%) (Fig. 1d). Compared to the control group and groups 1 and 2, differences reached statistical significance $(P<0.001)$.

The Ki-67 values ranged between $5-50 \%$ (on average $32 \%)$.

\section{Analysis of ER, PR, p53 and Ki-67 in simultaneously sampled eutopic endometrium}

Eutopic endometrium was available in 6 cases of DIE. When the immunophenotypes of simultaneously sampled eutopic endometrium and endometriotic foci were compared, there were 3 cases where differences only in the expression of Ki-67 were found; a significant decrease in endometriotic foci was found (17\% vs. $67 \%$ ).

\section{DISCUSSION}

In our study, we retrospectively reviewed a series of 120 cases of ovarian endometriomas; the diagnosis of atypical endometriosis was determined in 5 (4.2\%). In all these cases, the criteria described by LaGrenade and Silverberg ${ }^{15}$ and Czernobilsky and Morris ${ }^{16}$ were strictly followed for diagnosis. However, despite these histological criteria, severe epithelial atypia may be of reactive origin and the distinction is therefore problematic and limited. Some studies concluded that atypical endometriosis is not a result of reactive inflammation but rather possesses a precancerous potential because epithelial atypia occur irrespective of severe stromal inflammation, and are con- sidered the precursor lesion for endometriosis associated carcinomas (most commonly clear cell or endometrioid). None of our cases of atypical endometriosis exhibited more than a scant inflammatory infiltrate (mostly represented by rare intraepithelial neutrophils). The endometrioid epithelium was focally denuded and, in some cases, it was problematic to distinguish between moderate (reactive) and severe celular atypia. In these cases, we used a simple immunohistochemical panel with antibodies against ER, PR, p53 and Ki-67. The immunophenotype of endometriotic lesions with severe celular atypia was as follows: significantly higher strong p53 expression and lower ER and PR expression with only moderate staining. The immunophenotype of cases with reactive (moderate) atypia as well as the $\mathrm{Ki}-67$ values were comparable to nonatypical endometriotic lesions. Based on these findings, all ovarian endometriotic lesions with such an immunophenotype and severe celular atypia were classified as atypical endometriosis. A significant decrease in receptor content indicates a greater degree of autonomy of atypical endometriotic lesions. These findings, together with a strong and significantly higher p53 expression support the preneoplastic potential of the atypical endometriotic tissue. Although the diagnosis of atypical endometriosis should be based primarily on morphology, finding of such an immunophenotype (in the appropriate morphological context) is a very useful ancillary method.

Diagnosis of atypical endometriosis should be followed by careful long-term observation of the patient; the reason is the possible subsequent development of neoplasia. When searching the literature, the incidence of atypical endometriosis in ovarian cancer ranges from 4.4 to $22.8 \%$ and without neoplasm involvement from 1.7 to $32.3 \%$ (ref. ${ }^{18-21}$ ). At our hospital, the frequency of atypical endometriosis was $4.2 \%$ between 2016-2018; so far no neoplasm has developed in any of these cases.

The possibility of malignant transformation of endometriosis was originally mentioned by Samson in 1925 (ref. ${ }^{22}$ ) and later by Scott in 1953 (ref. ${ }^{23}$ ). However, the association between atypical endometriosis and ovarian neoplasm was originally described by LaGrenade and Silverberg in 1988 - clear cell carcinoma and endometrioid carcinoma have been found in continuity with atypical ovarian endometriosis ${ }^{15}$.

A number of studies immunohistochemically analyzed the ER and PR expression in endometrial tissue. In the study of Bur et al., it was conluded that ER expression in most endometriotic lesions corresponds to their morphological appearance ${ }^{24}$. These results are fully consistent with the results of the presented study. In another study, Lessey et al. found that receptor content in endometriotic tissue was more heterogeneous, unlike eutopic endometrium, and did not undergo predictable changes in response to endogenous hormones. The authors also described a significant decrease in ER and PR in both the glands and stroma in the endometriotic tissue of patients treated with hormonal therapy relative to untreated patients $^{25}$. In our series, 8 women took progestins. In 2 of them progestin-mediated morphologic changes in endometriotic tissue were observed (atrophic pattern with 
weak secretory changes). In these two cases, loss of PR expression (only in glands) and a slight decrease in ER expression (in both stroma and glands) were noted. The histologic appearance of the remaining 6 cases was inactive. In general, the morphological appearance of the eutopic endometrium and endometriotic tissue after progestin treatment is influenced by several factors, in particular the dose and duration of hormonal treatment, as well as the status of the endometrium ${ }^{26}$.

Some studies describe an overall reduction in PR and ER expression in endometriotic lesions relative to eutopic endometrium ${ }^{27,28}$. We observed this finding only in isolated cases. PR expression decreases significantly in the mid and late secretory phase in both endometriotic tissue and eutopic endometrium. Lower concentrations of PR receptors were observed in only 2 cases of DIE with early proliferative and inactive appearance, but not in LNE. In terms of oestrogen receptors, significant differences were found in only one case of DIE with inactive appearance, compared to LNE, where all cases showed strong ER expression in more than $90 \%$ of both glandular and stromal cells.

In our study, considerable attention was paid to the analysis of PNI. We found a total of 5 cases of the nerve involvement due to endometriosis. Regardless of the anatomical location of PNI, significant changes in the affected nerve tissue were detected. We introduced the term ,endometriotic neuropathy“, which is defined by distinct neuronal hypertrophy and hyperplasia with concomitant involvement of the ganglia of the autonomic nervous system (ANS) located in the wall of the affected organs as well as in the adjacent fibroadipose tissue. These ganglia, consisting of a population of both Schwann and ganglion cells, were easily detectable on the tissue sections stained with hematoxylin-eosin. Immunohistochemically, neuralappearing bundles and ganglia were strongly positive for S100 protein and neuron-specific enolase (NSE).

Regarding the organization of the ANS in the abdominopelvic organs, sympathetic presynaptic fibers lead from the lower thoracic and upper lumbar segments of the spinal cord via abdominopelvic splanchnic nerves to the vertebral and prevertebral ganglia, where postsynaptic neurons have their cell bodies. Parasymphatetic presynaptic neurons send axons from the sacral segments of the spinal cord to visceral ganglia that contain bodies of the postsynaptic neurons and are generally located in the walls of the organs, such as the ganglia of the submucosal (Meissner's) plexus, the deep submucosal (Henle's) plexus and the myenteric (Auerbach's) plexus in the alimentary canal ${ }^{29}$. It follows from the previous comments that, for all five of our cases, the ganglia affected by endometriosis are part of the parasympathetic branch of the ANS.

In general, the autonomic nervous system, which is subdivided into a sympathetic, parasymphatetic and enteric divisions, conducts impulses to smooth muscle, cardiac muscle, and glandular epithelium. The enteric division of the ANS controls motility, exocrine and endocrine secretions, and blood flow through the gastrointestinal tract. It also regulates immunologic and inflammatory processes. In addition to muscle fibers and the neural network, a population of the interstitial cells of Cajal (ICC) are critical for bowel motility and their function is modulated by both sympathetic and parasympathetic neural inputs ${ }^{30}$. When nerve fibers and ganglia of the ANS are affected by endometriosis, all of these functions can be impaired. As mentioned above, in our study PNI was a multifocal finding detected in the areas of macroscopically apparent lesions but also outside the confines of these areas. PNI allows the spread of endometriosis over a large distance and thus, PNI can make R0 resection of endometriotic lesions more difficult. These facts could be one of the causes of impaired functions of the affected organs after debulking surgery with macroscopic negative resection margins as well as pain symptomatology in macroscopic inapparent endometriotic lesions. Coversely, the absence of PNI could be the cause of the well-known situation of extensive organ involvement due to endometriosis and no pain at all.

\section{CONCLUSION}

From a histological and immunohistochemical point of view, deep infiltrating endometriosis and lymph node endometriosis appear to represent the same entity.

For the first time, a simple immunohistochemical panel with antibodies against ER, PR and p53 useful in diagnosing atypical endometriosis has been described.

The marked endometriosis-associated neural changes (endometriotic neuropathy) could be one of the causes of impaired functions of the affected organs after debulking surgery with macroscopic negative resection margins as well as pain symptomatology in macroscopic inapparent endometriotic lesions.

\section{ABBREVATIONS}

ANS, Autonomic nervous system; A, Atrophy; C, a combination of proliferative and secretory endometrium; DAB, Diaminobenzidine tetrahydrochloride; DIE, Deep infiltrating endometriosis; DNA, Deoxyribonucleic acid; EP, Early proliferative; ER, Oestrogen receptors; GnRH, Gonadotropin releasing hormone; ICC, Interstitial cells of Cajal; LNE, Lymph node endometriosis; LP, Late proliferative; LS, Late secretory; MP, Mid proliferative; MS, Mid secretory; NSE, Neuron-specific enolase; PNI, Perineural invasion; PR, Progesterone receptors; p53, Protein 53; rASRM, Revised American Society for reproductive medicine; RTU, Ready to use; USL, Uterosacral ligament.

Author contributions: JL: project development, manuscript writing; RCH: manuscript writing, critically revising the article; LF: statistical analysis, manuscript editing; PK, DL: manuscript writing, description of the histopathology findings, literature (PubMed) search.

Conflict of interest statement: The authors state that there 
are no conflicts of interest regarding the publication of this article.

\section{REFERENCES}

1. Johnson NP, Hummelshoj L. Consensus on current management of endometriosis. World Endometriosis Society Montpellier Consortium. Hum Reprod 2013;28(6):1552-68.

2. Schenken RS, Guzick DS. Revised endometriosis classification: 1996. Fertil Steril 1997;67(5):815-16.

3. Johnson NP, Hummelshoj L, Adamson GD, Keckstein J, Taylor HS Abrao MS, Bush D, Kiesel L, Tamimi R, Sharpe-Timms KL, Rombauts L, Giudice LC. World Endometriosis Society consensus on the classification of endometriosis. World Endometriosis Society Sao Paulo Consortium. Hum Reprod 2017;32(2):315-24.

4. Lindheim SR, Glenn T, Gagneux P, Maxwell RA, Yaklic JL, Findley $A D$, Bhagavath B. Current Challenges in the Diagnosis of Deep Infiltrating Endometriosis. Androl Gynecol 2018. Curr Res 6:1.

5. Thomas EJ, Campbell IG. Molecular genetic defects in endometriosis Gynecol Obstet Incest 2000;50 Suppl 1:44-50.

6. Insabato L, Pettinato G. Endometriosis of the bowel with lymph node involvement. A report of three cases and review of the literature. Pathol Res Pract 1996;192(9):957-61.

7. Mechsner S, Weichbrodt M, Riedlinger WF, Kaufmann AM, Schneider A, Köhler C. Immunohistochemical evaluation of endometriotic lesions and disseminated endometriosis-like cells in incidental lymph nodes of patients with endometriosis. Fertil Steril 2010;94(2):457-63.

8. Noël JC, Chapron C, Fayt I, Anaf V. Lymph node involvement and lymphovascular invasion in deep infiltrating rectosigmoid endometriosis. Fertil Steril 2008;89(5):1069-72.

9. McCluggage WG, Bryson C, Lamki H, Boyle DD. Benign, borderline, and malignant endometrioid neoplasia arising in endometriosis in association with tamoxifen therapy. Int J Gynecol Pathol 2000;19(3):276-9.

10. Kitawaki J, Kado N, Ishihara H, Koshiba H, Kitaoka $\mathrm{Y}$, Honjo H. Endometriosis: the pathophysiology as an estrogen-dependent disease. J Steroid Biochem Mol Biol 2002;83(1-5):149-55.

11. Giudice LC. Clinical practice. Endometriosis. N Engl J Med. 2010;362(25):2389-98

12. Ozaki T, Nakagawara A. p53: the attractive tumor suppressor in the cancer research field. J Biomed Biotechnol 2011;2011:603925.

13. Yan HL, Xue G, Mei Q, Wang YZ, Ding FX, Liu MF, Lu MH, Tang Y, Yu HY, Sun SH. Repression of the miR-17-92 cluster by p53 has an important function in hypoxia-induced apoptosis. EMBO J 2009;28(18):2719 32.
14. Engeland K. Cell cycle arrest through indirect transcriptional repression by p53: I have a DREAM. Cell Death Differ 2018;25(1):114-32.

15. LaGrenade A, Silverberg SG. Ovarian tumors associated with atypical endometriosis. Hum Pathol 1988;19(9):1080-84.

16. Czernobilsky B, Morris WJ. A histologic study of ovarian endometriosis with emphasis on hyperplastic and atypical changes. Obstet Gynecol 1979;53(3):318-23.

17. Noyes FW, Hertig AT, Rock J. Dating the endometrial biopsy. Fertil Steril 1950;1:3-25.

18. Fukunaga $M$, Nomura $K$, Ishikawa $E$, Ushigome $S$. Ovarian atypica endometriosis: its close association with malignant epithelial tumors. Histopathology 1997;30(3):249-55.

19. Seidman JD. Prognostic importance of hyperplasia and atypia in endometriosis. Int J Gynecol Pathol 1996;15(1):1-9.

20. Bayramoglu H, Duzcan E. Atypical epithelial change and mutant p53 gene expression in ovarian endometriosis. Pathol Oncol Res 2001;7:33-8.

21. Oral E, Ilvan S, Tustas E, Korbeyli B, Bese T, Demirkiran F, Arvas M, Kosebay D. Prevalence of endometriosis in malignant epithelial ovary tumours. Eur J Obstet Gynecol Reprod Biol 2003;109(1):97-101.

22. Sampson JA. Endometrial carcinoma of the ovary arising in endometrial tissue in that organ. Arch Surg 1925;10:1.

23. Scott RB. Malignant changes in endometriosis. Obstet Gynecol 1953;2:283.

24. Bur ME, Greene GL, Press MF. Estrogen receptor localization in formalin-fixed, paraffin-embedded endometrium and endometriotic tissues. Int J Gynecol Pathol 1987;6(2):140-51.

25. Lessey BA, Metzger DA, Haney AF, McCarty KS Jr. Immunohistochemical analysis of estrogen and progesterone receptors in endometriosis: comparison with normal endometrium during the menstrual cycle and the effect of medical therapy. Fertil Steril 1989;51(3):409-15.

26. Deligdisch L. Hormonal pathology of the endometrium. Mod Pathol 2000;13(3):285-94.

27. Attia GR, Zeitoun K, Edwards D, Johns A, Carr BR, Bulun SE. Progesterone receptor isoform $A$ but not $B$ is expressed in endometriosis. J Clin Endocrinol Metab 2000;85(8):2897-902.

28. Burney RO, Talbi S, Hamilton AE, Vo KC, Nyegaard M, Nezhat CR Lessey BA, Giudice LC. Gene expression analysis of endometrium reveals progesterone resistance and candidate susceptibility genes in women with endometriosis. Endokrinology 2007;148(8):3814-26.

29. Ross MH, Pawlina W. Histology: A text and atlas with correlated cell and molecular biology. 5th ed. Philadelphia: Lippincott Williams and Wilkins; 2005.

30. Karemaker JM. An introduction into autonomic nervous function Physiol Meas 2017;38(5):R89-R118. 